Courrier du Centre international Blaise Pascal

$4 \mid 1982$

Varia

\title{
Les progrès et les besoins du CIBP
}

Thérèse Goyet

\section{OpenEdition}

Journals

Édition électronique

URL : http://journals.openedition.org/ccibp/406

DOI : $10.4000 /$ ccibp. 406

ISSN : 2493-7460

\section{Éditeur}

Centre international Blaise Pascal

\section{Édition imprimée}

Date de publication : 4 mars 1982

Pagination : 14

ISSN : 0249-6674

\section{Référence électronique}

Thérèse Goyet, "Les progrès et les besoins du CIBP », Courrier du Centre international Blaise Pascal [En ligne], 4 | 1982, mis en ligne le 25 novembre 2015, consulté le 19 avril 2019. URL : http:// journals.openedition.org/ccibp/406 ; DOI : 10.4000/ccibp.406

Ce document a été généré automatiquement le 19 avril 2019.

Centre international Blaise Pascal 


\title{
Les progrès et les besoins du CIBP
}

\author{
Thérèse Goyet
}

\section{Blaise Pascal, un « Auvergnat » dans la vallée de Chevreuse}

1 La Maison des Jeunes et de la Culture de Gif-sur-Yvette organise, avec l'aide de certaines associations et des collectivités locales de toute la vallée de Chevreuse, une animation très variée ayant pour titre « Port-Royal et son temps » (28 mai-12 juin 1983).

2 Pour l'exposition «Blaise Pascal » qui se tiendra à la Salle de la Terrasse à Gif-sur-Yvette, le CIBP a prêté le matériel scientifique des expériences qui ont été présentées à l'exposition de Clermont en 1981.

\section{Publications en dépôt au CIBP}

3 Vous pouvez demander :

- Le catalogue de l'exposition Blaise Pascal « auvergnat ». Clermont-Ferrand, 1981, 110 p. 18 pl. fig. $40 \mathrm{~F}+$ port $8,50 \mathrm{~F}$.

- Quatre cartes postales reproduisant les filigranes du papier utilisé par Pascal. $10 \mathrm{~F}+$ port 2,90 F

- Deux grandes figures d'Auvergne, Gilberte et Jacqueline Pascal. Chroniques de Port-Royal, $\mathrm{n}^{\circ} 31$. Clermont Ferrand, 1982. 158 p. 9 pl. -120 F franco.

\section{Acquisition désirées}

4 La Bibliothèque Municipale et Interuniversitaire de Clermont-Ferrand ne possède pas l'édition Bossut des CEuvres de Blaise Pascal, La Haye : Detune, 1779.5 vol. in- $8^{\circ}$, les éditions Renouard des Pensées, 2 vol. in- $8^{\circ}, 1803$ et 1812 Provinciales, 2 vol. in-12, 1803.

5 Le CIBP serait très reconnaissant aux personnes qui lui permettraient de les acquérir. 


\section{Liste des dons reçus par le CIBP durant le second trimestre 1982 et le premier trimestre 1983}

\section{Livres, articles et revues}

Brises (Bulletin de Recherches sur l'Information en Sciences économiques, humaines et sociales). Paris : C.D.S.H., $1982 . \mathrm{N}^{\circ} 1$.

Documents pour l'histoire du vocabulaire scientifique. Paris : Institut national de la langue française (CNRS), 1980. $\mathrm{N}^{\circ} 1$ à 4.

FRANCOVICH Guillermo. Ensayos pascalianos. Sucre (Bolivie) : Division de extension universitaria, 1979. $173 \mathrm{p}$.

HARRINGTON Thomas More. Pascal philosophe : une étude unitaire de la pensée de Pascal. Paris : C.D.U. et SEDES, 1982. $187 \mathrm{p}$.

MEURILLON Christian. « La Machine arithmétique à la genèse des ordres pascaliens. » In : La Machine dans l'imaginaire, Revue des sciences humaines, 58, 186-187, 1982-1983.

MEURILLON Christian. « Saint-Réal et Pascal. » In : SAINTRÉAL (César Vichard, abbé de). De l'Usage de l'histoire. Villeneuve d'Ascq : Université de Lille, 1980.

PASCAL Blaise. Les Pensées de Pascal ;éditées par Francis Kaplan. Paris : Éd. du Cerf, 1982. 705 p.

SCHMITZ DU MOULIN Henri. Blaise Pascal : une biographie spirituelle. Assen (Pays-Bas) Van Gorcum, 1982. - XII. 150 p.

SELLIER Philippe. « Jésus-Christ chez Pascal. » Paris : Revue des sciences philosophiques et théologiques, 66, 4, 1982. $18 \mathrm{p}$.

SOVERAL Edouardo Abranches de. Pascal filosofo cristâo. Porto : Tavares Martins, 1968. - 294 p.

THIROUIN Laurent. - Raison des effets : essai d'explication d'un concept pascalien. - Paris : Dixseptième siècle, $1,134,1982$. -. 20 p.

TOURNEUR Zacharie. - Beauté poétique : histoire critique d'une «Pensée » de Pascal... Melun : R. Rozelle, 1933. $166 \mathrm{p}$.

\section{Documents divers}

PASCAL Blaise. Lettres d'A. Dettonville. Clermont Ferrand : Facultés des Lettres, 1981. 1 microfilm. (Microéd. de l'exemplaire des Archives de l'Académie des Sciences, Paris, 1659)

PASCAL Blaise. Lettres d'A. Dettonville. Clermont Ferrand : Faculté des Lettres, 1981. 18 photos. Blaise Pascal ou le plaisir aux catacombes : texte du spectacle proposé par le Théâtre de la Fontanelle. Paris, 1981. 42 f. dactyl. 
INDEX

Mots-clés : CIBP

\section{AUTEUR}

THÉRÈSE GOYET

Professeur à l'Université II de Clermont-Ferrand 